\title{
$\Delta$ \\ A note on generators of the endomorphism semigroup of an infinite countable chain
}

\author{
Ilinka Dimitrova, Vítor H. Fernandes* and Jörg Koppitz
}

October 17, 2018

\begin{abstract}
In this note, we consider the semigroup $\mathcal{O}(X)$ of all order endomorphisms of an infinite chain $X$ and the subset $J$ of $\mathcal{O}(X)$ of all transformations $\alpha$ such that $|\operatorname{Im}(\alpha)|=|X|$. For an infinite countable chain $X$, we give a necessary and sufficient condition on $X$ for $\mathcal{O}(X)=\langle J\rangle$ to hold. We also present a sufficient condition on $X$ for $\mathcal{O}(X)=\langle J\rangle$ to hold, for an arbitrary infinite chain $X$.
\end{abstract}

2010 Mathematics subject classification: 20M20, 20M10.

Keywords: infinite chain, endomorphism semigroup, generators, relative rank.

\section{Introduction}

The rank of a semigroup $S$ is the minimum cardinality of a generating set of $S$. For a countable semigroup $S$, in particular, for a finitely generated semigroup $S$, determining the rank of $S$ is a natural question. Contrariwise, for an uncountable semigroup $S$, this concept has no interest, since the rank of $S$ is always $|S|$. This last fact leads to the following notion. For a subset $A$ of a semigroup $S$, the relative rank of $S$ modulo $A$ is the minimum cardinality of a subset $B$ of $S$ such that $\langle A \cup B\rangle=S$. This cardinal is denoted by $\operatorname{rank}(S: A)$. It follows immediately from the definition that $\operatorname{rank}(S: A)=\operatorname{rank}(S:\langle A\rangle)$ and that $\operatorname{rank}(S: A)=0$ if and only if $A$ is a generating set of $S$.

${ }^{*}$ This work was developed within the FCT Project UID/MAT/00297/2013 of CMA and of Departamento de Matemática da Faculdade de Ciências e Tecnologia da Universidade Nova de Lisboa. 
The notion of relative rank was introduced by Ruškuc in [8], who proved that the rank of a finite Rees matrix semigroup $\mathcal{M}[G ; I, \Lambda ; P]$, with the sandwich matrix $P$ in normal form, is equal to $\max \{|I|,|\Lambda|, \operatorname{rank}(G: H)\}$, where $H$ is the subgroup of $G$ generated by the entries of $P$. In [6], Howie et al. considered the relative ranks of the full transformation semigroup $\mathcal{T}(X)$ on $X$, where $X$ is an infinite set, modulo some distinguished subsets of $\mathcal{T}(X)$. They showed that $\operatorname{rank}(\mathcal{T}(X): \mathcal{S}(X))=2, \operatorname{rank}(\mathcal{T}(X): \mathcal{E}(X))=2$ and $\operatorname{rank}(\mathcal{T}(X): J)=0$, where $\mathcal{S}(X)$ is the symmetric group on $X, \mathcal{E}(X)$ is the set of all idempotent transformations on $X$ and $J$ is the top $\mathcal{J}$-class of $\mathcal{T}(X)$, i.e. $J=\{\alpha \in \mathcal{T}(X)|| \operatorname{Im}(\alpha)|=| X \mid\}$.

Throughout this paper, we will represent a chain only by its support set and, as usual, its order by the symbol $\leq$. Let $X$ be a chain. A transformation $\alpha$ of $X$ is said to be order-preserving or an (order) endomorphism of $X$ if $x \leq y$ implies $x \alpha \leq y \alpha$, for all $x, y \in X$. We denote by $\mathcal{O}(X)$ the subsemigroup of $\mathcal{T}(X)$ of all (order) endomorphisms of $X$.

For a finite chain $X$, it is well known, and clear, that $\mathcal{O}(X)$ is a regular semigroup. The problem for an infinite chain $X$ is much more involved. Nevertheless, more generally, a characterization of those posets $P$ for which the semigroup of all endomorphisms of $P$ is regular was done by Aizzenštat in 1968 [2] and, independently, by Adams and Gould in 1989 [1].

Let $X$ be an infinite chain. A useful regularity criterion for the elements of $\mathcal{O}(X)$ was proved in [7] by Mora and Kemprasit, who deduced several previous known results based on it: for instance, that $\mathcal{O}(\mathbb{Z})$ is regular while $\mathcal{O}(\mathbb{Q})$ and $\mathcal{O}(\mathbb{R})$ are not regular, by considering their usual orders. In [3, Fernandes et al. described the largest regular subsemigroup of $\mathcal{O}(X)$ and also Green's relations on $\mathcal{O}(X)$. The relative rank of $\mathcal{T}(X)$ modulo the subsemigroup $\mathcal{O}(X)$ was considered by Higgins et al. in [4]. They showed that $\operatorname{rank}(\mathcal{T}(X): \mathcal{O}(X))=1$, when $X$ is an arbitrary countable chain or an arbitrary well-ordered set, while $\operatorname{rank}(\mathcal{T}(\mathbb{R}): \mathcal{O}(\mathbb{R}))$ is uncountable, by considering the usual order of $\mathbb{R}$.

For a fixed chain $X$, consider the following two subsets of the semigroup $\mathcal{O}(X)$ :

$$
J=\{\alpha \in \mathcal{O}(X)|| \operatorname{Im}(\alpha)|=| X \mid\} \quad \text { and } \quad J_{f}=\left\{\alpha \in \mathcal{O}(X)|| \operatorname{Im}(\alpha) \mid<\aleph_{0}\right\} .
$$

Notice that $J_{f}$ is clearly an ideal of $\mathcal{O}(X)$. On the other hand, unlike the analogous set for $\mathcal{T}(X), J$ is not necessarily a $\mathcal{J}$-class of $\mathcal{O}(X)$ (see [3]).

In this note we study the relative rank of the semigroup $\mathcal{O}(X)$ modulo $J$. For an infinite countable chain $X$, we give a necessary and sufficient condition on $X$ for $\mathcal{O}(X)=\langle J\rangle$ to hold (notice that, for a finite $X, \mathcal{O}(X)=$ 
$\langle J\rangle$ if and only if $|X|=1$ ). We also present a sufficient condition on $X$ for $\mathcal{O}(X)=\langle J\rangle$ to hold, for an arbitrary infinite chain $X$.

For general background on Semigroup Theory, we refer the reader to Howie's book [5].

\section{Main results}

Let $X$ be an infinite chain. Let $x \in X$ and define

$$
(x]=\{y \in X \mid y \leq x\} \quad \text { and } \quad[x)=\{y \in X \mid x \leq y\}
$$

(i.e the left and right order ideals generated by $x$ ). Define also

$$
\begin{gathered}
X^{0}=\{x \in X||(x]|=| X|=|[x) \mid\}, \\
X^{-}=\{x \in X||(x]|<| X \mid\}
\end{gathered}
$$

and

$$
X^{+}=\{x \in X||[x)|<| X \mid\} .
$$

Notice that, since $X$ is an infinite set, if $x \in X^{-}$(respectively, $x \in X^{+}$) then $|X|=|[x)|$ (respectively, $|X|=|(x]|)$. Hence $X$ is a disjoint union of $X^{-}, X^{0}$ and $X^{+}$.

Let us consider the sets $\mathbb{N}, \mathbb{Z}_{-}=\mathbb{Z} \backslash(\mathbb{N} \cup\{0\}), \mathbb{Z}, \mathbb{Q}$ and $\mathbb{R}$, with their usual orders. Then, we have:

1. $X^{-}=\mathbb{N}, X^{0}=\emptyset$ and $X^{+}=\emptyset$, if $X=\mathbb{N}$;

2. $X^{-}=\emptyset, X^{0}=\emptyset$ and $X^{+}=\mathbb{Z}_{-}$, if $X=\mathbb{Z}_{-}$;

3. $X^{-}=\emptyset, X^{0}=X$ and $X^{+}=\emptyset$, for $X \in\{\mathbb{Z}, \mathbb{Q}, \mathbb{R}\}$.

Recall that, given two posets $P$ and $Q$ with disjoint supports, the ordinal sum $P \oplus Q$ of $P$ and $Q$ (by this order) is the poset with support $P \cup Q$ such that $P$ and $Q$ are subposets of $P \oplus Q$ and $x<y$, for all $x \in P$ and $y \in Q$. This operation on posets is associative (but not commutative). For our purposes, it is convenient to admit empty posets.

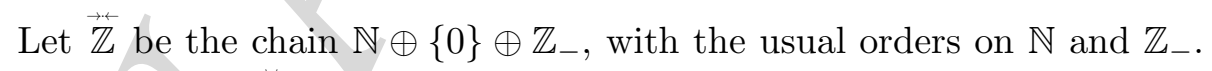
Then, being $X=\overrightarrow{\mathbb{Z}}$, we have $X^{-}=\mathbb{N}, X^{0}=\{0\}$ and $X^{+}=\mathbb{Z}_{-}$.

By considering $X^{-}, X^{0}$ and $X^{+}$as subposets of $X$, we have the following decomposition of $X$ : 
Lemma 1.1. Let $X$ be an infinite chain. Then $X=X^{-} \oplus X^{0} \oplus X^{+}$.

Proof. First, let $a \in X^{-}$and $b \in X^{0} \cup X^{+}$. If $b \leq a$ then $(b] \subseteq(a]$ and so $|X|=|(b]| \leq|(a]|<|X|$, a contradiction. Then $a<b$. On the other hand, given $a \in X^{-} \cup X^{0}$ and $b \in X^{+}$, by a dual reasoning, we may show that $a<b$. This proves the lemma.

Note 1.2. Let $X$ be an infinite chain and let $\alpha \in \mathcal{O}(X)$. If there exist $x^{+} \in X^{+}$and $x^{-} \in X^{-}$such that $x^{+} \alpha=x^{-}$or $x^{-} \alpha=x^{+}$then $\alpha \notin J$.

In fact, suppose that $x^{+} \alpha=x^{-}$(the other case can be treated dually). Then $\operatorname{Im}(\alpha) \subseteq\left(x^{-}\right] \cup\left[x^{+}\right) \alpha$ and so $|\operatorname{Im}(\alpha)| \leq\left|\left(x^{-}\right]\right|+\left|\left[x^{+}\right) \alpha\right| \leq\left|\left(x^{-}\right]\right|+$ $\left|\left[x^{+}\right)\right|<|X|+|X|=|X|$, i.e. $\alpha \notin J$, as required.

Note 1.3. Let $X$ be an infinite chain such that $X^{0}=\emptyset$. Let $\alpha \in \mathcal{O}(X)$ be such that $x^{+} \alpha=x^{-}$or $x^{-} \alpha=x^{+}$, for some $x^{+} \in X^{+}$and $x^{-} \in X^{-}$. Let $\alpha_{1}, \alpha_{2}, \ldots, \alpha_{n} \in \mathcal{O}(X)$ be such that $\alpha=\alpha_{1} \alpha_{2} \cdots \alpha_{n}$. Then $|\operatorname{Im}(\alpha)| \leq$ $\left|\operatorname{Im}\left(\alpha_{i}\right)\right|<|X|$ (and so $\left.\alpha, \alpha_{i} \notin J\right)$, for some $i=1,2, \ldots, n$.

In fact, for the case $x^{+} \alpha=x^{-}$(the other case is dual), let $i=\min \{j \in$ $\left.\{1, \ldots, n\} \mid x^{+} \alpha_{1} \cdots \alpha_{j} \in X^{-}\right\}$. Then $x^{+} \alpha_{1} \cdots \alpha_{i-1} \in X^{+}$(for $i=1$ the expression $x^{+} \alpha_{1} \cdots \alpha_{i-1}$ has the meaning of $\left.x^{+}\right)$and $\left(x^{+} \alpha_{1} \cdots \alpha_{i-1}\right) \alpha_{i} \in X^{-}$, since $X^{0}=\emptyset$. Hence, by Note $1.2, \alpha_{i} \notin J$. On the other hand, from the equality $\alpha=\alpha_{1} \alpha_{2} \cdots \alpha_{n}$, it follows that $|\operatorname{Im}(\alpha)| \leq\left|\operatorname{Im}\left(\alpha_{j}\right)\right|$ (indeed $\operatorname{Im}\left(\alpha_{1} \cdots \alpha_{j}\right) \subseteq \operatorname{Im}\left(\alpha_{j}\right)$, whence $\operatorname{Im}(\alpha)=\left(\operatorname{Im}\left(\alpha_{1} \cdots \alpha_{j}\right)\right)\left(\alpha_{j+1} \cdots \alpha_{n}\right) \subseteq$ $\left(\operatorname{Im}\left(\alpha_{j}\right)\right)\left(\alpha_{j+1} \cdots \alpha_{n}\right)$ and so $\left.|\operatorname{Im}(\alpha)| \leq\left|\left(\operatorname{Im}\left(\alpha_{j}\right)\right)\left(\alpha_{j+1} \cdots \alpha_{n}\right)\right| \leq\left|\operatorname{Im}\left(\alpha_{j}\right)\right|\right)$, for all $j \in\{1, \ldots, n\}$, and so $|\operatorname{Im}(\alpha)| \leq\left|\operatorname{Im}\left(\alpha_{i}\right)\right|<|X|$, as required.

This last note can be rewritten as follows:

Lemma 1.4. Let $X$ be an infinite chain such that $X^{0}=\emptyset$ and let $\alpha \in \mathcal{O}(X)$ be such that $X^{+} \alpha \cap X^{-} \neq \emptyset$ or $X^{-} \alpha \cap X^{+} \neq \emptyset$. Then $\alpha \notin\langle J\rangle$.

Before presenting our next note, we introduce the following (natural) notation. For $x \in X$ and $Y \subseteq X$, by $x<Y$ (respectively, $x>Y$ ) we mean that $x<y$ (respectively, $x>y$ ), for all $y \in Y$.

Note 1.5. Let $X$ be an infinite chain and let $\alpha \in \mathcal{O}(X)$.

1. If $b \in \operatorname{Im}(\alpha)$ and there exists no element $c \in X$ such that $c<b \alpha^{-1}$ then $\operatorname{Im}(\alpha) \subseteq[b)$.

In fact, let $y \in \operatorname{Im}(\alpha)$. Take $x \in y \alpha^{-1}$. Then $x \nless b \alpha^{-1}$ and so there exists $a \in b \alpha^{-1}$ such that $a \leq x$. It follows that $b=a \alpha \leq x \alpha=y$, whence $y \in[b)$, as required. 
2. If $\alpha \in J$ and $b \in \operatorname{Im}(\alpha) \cap X^{+}$then there exists an element $c \in X$ such that $c<b \alpha^{-1}$.

In fact, if there exists no element $c \in X$ such that $c<b \alpha^{-1}$ then, by 1 above, we have $\operatorname{Im}(\alpha) \subseteq[b)$ and, as $b \in X^{+}$, it follows $|\operatorname{Im}(\alpha)| \leq$ $|[b)|<|X|$, whence $\alpha \notin J$, a contradiction.

3. If $\alpha \in J$ and $y \in X^{+}$then there exists an element $b \in \operatorname{Im}(\alpha)$ such that $b<y$.

In fact, if $y \leq b$, for all $b \in \operatorname{Im}(\alpha)$, then $\operatorname{Im}(\alpha) \subseteq[y)$ and, as $y \in X^{+}$, it follows $|\operatorname{Im}(\alpha)| \leq|[y)|<|X|$, whence $\alpha \notin J$, a contradiction.

By combining 2 and 3 of the previous note, it follows immediately:

Note 1.6. Let $X$ be an infinite chain such that $X=X^{+}$, let $\alpha, \beta \in J$ and let $b \in \operatorname{Im}(\alpha)$. Then there exist $c \in X$ and $b^{\prime} \in \operatorname{Im}(\beta)$ such that $b^{\prime}<c<b \alpha^{-1}$.

From 3 of Note 1.5, if $X=X^{+}$, it is clear that $\operatorname{Im}(\alpha)$ has no lower bounds, for all $\alpha \in J$. Moreover, we have:

Lemma 1.7. Let $X$ be an infinite chain such that $X=X^{+}$(respectively, $X=X^{-}$) and let $\alpha \in\langle J\rangle$. Then $\operatorname{Im}(\alpha)$ has no minimum (respectively, maximum). In particular $J_{f} \cap\langle J\rangle=\emptyset$.

Proof. We prove this result for $X=X^{+}$. The case $X=X^{-}$is dual.

By contradiction, let us suppose that $\operatorname{Im}(\alpha)$ has minimum. Denote $\min \operatorname{Im}(\alpha)$ by $b_{n}$.

As $\alpha \in\langle J\rangle$, we have $\alpha=\alpha_{1} \alpha_{2} \cdots \alpha_{n}$, for some $\alpha_{1}, \alpha_{2}, \ldots, \alpha_{n} \in J$.

Notice that, since $b_{n} \in \operatorname{Im}(\alpha)$, we also have $b_{n} \in \operatorname{Im}\left(\alpha_{n}\right)$. By applying Note 1.6. we find elements $c_{n} \in X$ and $b_{n-1} \in \operatorname{Im}\left(\alpha_{n-1}\right)$ such that

$$
b_{n-1}<c_{n}<b_{n} \alpha_{n}^{-1} .
$$

By applying again Note 1.6, we can take elements $c_{n-1} \in X$ and $b_{n-2} \in$ $\operatorname{Im}\left(\alpha_{n-2}\right)$ such that

$$
b_{n-2}<c_{n-1}<b_{n-1} \alpha_{n-1}^{-1} .
$$

Moreover, by Note 1.6, we may recursively construct two sequences

$$
c_{n}, c_{n-1}, \ldots, c_{2} \quad \text { and } \quad b_{n-1}, b_{n-2}, \ldots, b_{1}
$$

of elements of $X$ such that $b_{i-1} \in \operatorname{Im}\left(\alpha_{i-1}\right)$ and

$$
b_{i-1}<c_{i}<b_{i} \alpha_{i}^{-1}
$$


for $i=2, \ldots, n$. In addition, by Note 1.5, we may also consider an element $c_{1} \in X$ such that $c_{1}<b_{1} \alpha_{1}^{-1}$.

Let $i=1,2, \ldots, n$. Then, $c_{i} \alpha_{i}<b_{i}$. In fact, since $c_{i}<b_{i} \alpha_{i}^{-1}$, we get $c_{i} \notin b_{i} \alpha_{i}^{-1}$, whence $c_{i} \alpha_{i} \neq b_{i}$, and, given $a \in b_{i} \alpha_{i}^{-1}$, we have $c_{i}<a$ and so $c_{i} \alpha_{i} \leq a \alpha_{i}=b_{i}$.

Next, by induction on $i$, we prove that $c_{1} \alpha_{1} \alpha_{2} \cdots \alpha_{i}<b_{i}$, for $i=$ $1,2, \ldots, n$. Let $i=1$. Then, the inequality $c_{1} \alpha_{1}<b_{1}$ was already proved above. Hence, let $i>1$ and suppose that $c_{1} \alpha_{1} \alpha_{2} \cdots \alpha_{i-1}<b_{i-1}$, by induction hypothesis. Since $b_{i-1}<c_{i}$, we have $c_{1} \alpha_{1} \alpha_{2} \cdots \alpha_{i-1}<c_{i}$ and so $c_{1} \alpha_{1} \alpha_{2} \cdots \alpha_{i-1} \alpha_{i} \leq c_{i} \alpha_{i}<b_{i}$, as required.

Hence, in particular, we have $c_{1} \alpha=c_{1} \alpha_{1} \alpha_{2} \cdots \alpha_{n}<b_{n}=\min \operatorname{Im}(\alpha)$, which is a contradiction. Therefore, $\operatorname{Im}(\alpha)$ has no minimum, as required.

Next, we state our fundamental lemma.

Main Lemma. Let $X$ be an infinite chain. Then $J_{f} \subseteq\langle J\rangle$ if and only if $X^{0} \neq \emptyset$.

Proof. First, suppose that $X^{0}=\emptyset$. If $X^{+}=\emptyset$ or $X^{-}=\emptyset$ then, by Lemma 1.7, we have $J_{f} \cap\langle J\rangle=\emptyset$, whence $J_{f} \nsubseteq\langle J\rangle$ (notice that $J_{f} \neq \emptyset$ ). On the other hand, admit that $X^{+} \neq \emptyset$ and $X^{-} \neq \emptyset$. Fix $a \in X^{-}$and let $\alpha \in \mathcal{O}(X)$ be the constant transformation with image $\{a\}$. Then $X^{+} \alpha \cap X^{-} \neq \emptyset$ and so, by Lemma 1.4, $\alpha \notin\langle J\rangle$. Since $\alpha \in J_{f}$, in this case, we also obtain $J_{f} \not \subseteq\langle J\rangle$.

Conversely, suppose that $X^{0} \neq \emptyset$ and fix an element $0 \in X^{0}$. Let $\alpha \in J_{f}$.

Suppose, without loss of generality, that $0 \alpha \leq 0$ (the case $0 \alpha \geq 0$ can be treated dually).

We begin by defining a transformation $\beta \in \mathcal{O}(X)$ by

$$
x \beta= \begin{cases}x \alpha, & x \leq 0 \\ x, & x>0\end{cases}
$$

Next, let $\operatorname{Im}(\alpha)=\left\{a_{1}<a_{2}<\cdots<a_{n}\right\}, n \in \mathbb{N}$, and suppose that $0 \alpha=$ $a_{i}<a_{i+1}<\cdots<a_{i+k} \leq 0$, with $a_{i+k+1}>0$ or $i+k=n$, for some $i \in\{1, \ldots, n\}$ and a non-negative integer $k$. Then, for $0 \leq j \leq k$, we define transformations $\gamma_{1}^{(j)}$ and $\gamma_{2}^{(j)}$ of $\mathcal{O}(X)$ by

$x \gamma_{1}^{(j)}= \begin{cases}x, & x<0 \\ 0, & x \geq 0 \text { and } x \ngtr a_{i+j} \alpha^{-1} \text { and } x \gamma_{2}^{(j)}=\left\{\begin{array}{ll}x, & x<a_{i+j} \\ x, & x>a_{i+j} \alpha^{-1}\end{array} a_{i+j}, \quad a_{i+j} \leq x \leq 0\right. \\ x, & x>0 .\end{cases}$ 
Finally, we define a transformation $\delta \in \mathcal{O}(X)$ as being the identity map on $X$ if $i+k=n$ and by

$$
x \delta= \begin{cases}x, & x \leq 0 \\ a_{i+k+1}, & x>0 \text { and } x \ngtr a_{i+k+1} \alpha^{-1} \\ x \alpha, & x>a_{i+k+1} \alpha^{-1}\end{cases}
$$

otherwise.

Since $0 \in X^{0}$, it is clear that $\beta, \gamma_{1}^{(0)}, \gamma_{2}^{(0)}, \ldots, \gamma_{1}^{(k)}, \gamma_{2}^{(k)}, \delta \in J$. Moreover, we have $\alpha=\beta \gamma_{1}^{(0)} \gamma_{2}^{(0)} \cdots \gamma_{1}^{(k)} \gamma_{2}^{(k)} \delta$. In fact, taking $x \in X$, we may consider three cases:

1. If $x \leq 0$ then

$$
(x) \beta \gamma_{1}^{(0)} \gamma_{2}^{(0)} \cdots \gamma_{1}^{(k)} \gamma_{2}^{(k)} \delta=(x \alpha) \gamma_{1}^{(0)} \gamma_{2}^{(0)} \cdots \gamma_{1}^{(k)} \gamma_{2}^{(k)} \delta=x \alpha,
$$

since $x \alpha \leq a_{i}$

2. If $x>0$ and $x \alpha \leq 0$ then there exists $j \in\{0,1, \ldots, k\}$ such that $x \alpha=a_{i+j}$ and

$$
\begin{aligned}
(x) \beta \gamma_{1}^{(0)} \gamma_{2}^{(0)} \cdots \gamma_{1}^{(k)} \gamma_{2}^{(k)} \delta & =(x) \gamma_{1}^{(j)} \gamma_{2}^{(j)} \cdots \gamma_{1}^{(k)} \gamma_{2}^{(k)} \delta \\
& =(0) \gamma_{2}^{(j)} \cdots \gamma_{1}^{(k)} \gamma_{2}^{(k)} \delta \\
& =\left(a_{i+j}\right) \gamma_{1}^{(j+1)} \gamma_{2}^{(j+1)} \cdots \gamma_{1}^{(k)} \gamma_{2}^{(k)} \delta \\
& =a_{i+j} \\
& =x \alpha
\end{aligned}
$$

3. If $x>0$ and $x \alpha>0$ then

$$
(x) \beta \gamma_{1}^{(0)} \gamma_{2}^{(0)} \cdots \gamma_{1}^{(k)} \gamma_{2}^{(k)} \delta=x \delta=x \alpha .
$$

Thus $\alpha \in\langle J\rangle$ and so $J_{f} \subseteq\langle J\rangle$, as required.

The following observation will be useful in the proof of our next result.

Note 1.8. Let $X$ be an infinite chain. Then $J_{f}$ contains elements of arbitrary finite (non null) rank. In fact, for all $n \in \mathbb{N}$ and $x_{1}, x_{2}, \ldots, x_{n} \in X$, with $x_{1}<x_{2}<\cdots<x_{n}$, we may construct transformations $\alpha \in \mathcal{O}(X)$ such that $\operatorname{Im}(\alpha)=\left\{x_{1}, x_{2}, \ldots, x_{n}\right\}$. For instance, the transformation $\alpha$ on $X$ defined by

belongs to $J_{f}$.

$$
x \alpha= \begin{cases}x_{1}, & x \leq x_{1} \\ x_{i}, & x_{i-1}<x \leq x_{i} \text { and } 2 \leq i \leq n-1 \\ x_{n}, & x_{n-1}<x\end{cases}
$$


Notice that if $X$ is an infinite countable chain then $J_{f}=\mathcal{O}(X) \backslash J$. Thus, in this case, by the previous lemma, we obtain that $\mathcal{O}(X)=\langle J\rangle$ if and only if $X^{0} \neq \emptyset$. Furthermore, we have:

Theorem 1.9. Let $X$ be an infinite countable chain. The following properties are equivalent:

1. $\mathcal{O}(X)=\langle J\rangle$, i.e. $\operatorname{rank}(\mathcal{O}(X): J)=0$;

2. $\operatorname{rank}(\mathcal{O}(X): J)<\aleph_{0}$;

3. $X^{0} \neq \emptyset$.

Proof. Notice that 1 trivially implies 2 and, by the previous lemma, 3 implies 1 , whence it remains to prove that 2 implies 3 . Thus, suppose that $X^{0}=\emptyset$. Let $\mathcal{C}$ be a generating set of $\mathcal{O}(X)$.

First, we admit that $X^{-} \neq \emptyset$ and $X^{+} \neq \emptyset$. As $X^{0}=\emptyset$, we must have $\left|X^{-}\right|=\aleph_{0}$ or $\left|X^{+}\right|=\aleph_{0}$. Suppose, without loss of generality, that $\left|X^{-}\right|=\aleph_{0}$ (the case $\left|X^{+}\right|=\aleph_{0}$ can be treated dually). Hence, given $n \in \mathbb{N}$, we may consider $n$ elements $x_{1}, x_{2}, \ldots, x_{n} \in X^{-}$, with $x_{1}<x_{2}<\cdots<x_{n}$, and the transformation $\alpha \in \mathcal{O}(X)$ such that $\operatorname{Im}(\alpha)=\left\{x_{1}, x_{2}, \ldots, x_{n}\right\}$ constructed in Note 1.8. Then, for any $x^{+} \in X^{+}$, we have $x^{+} \alpha=x_{n} \in X^{-}$and so, accordingly with Note 1.3, $\mathcal{C}$ contains a transformation $\beta \in \mathcal{O}(X)$ such that $n=|\operatorname{Im}(\alpha)| \leq|\operatorname{Im}(\beta)|<\aleph_{0}$. Thus, as $n \in \mathbb{N}$ is arbitrary, $\mathcal{C}$ must contain an infinite number of elements of $J_{f}$.

On the other hand, admit that $X^{+}=\emptyset$ or $X^{-}=\emptyset$. Then, by Lemma 1.7. we have $J_{f} \cap\langle J\rangle=\emptyset$. Let $n \in \mathbb{N}$, let $x_{1}, x_{2}, \ldots, x_{n} \in X$ be such $x_{1}<x_{2}<\cdots<x_{n}$ and consider the transformation $\alpha \in \mathcal{O}(X)$ such that $\operatorname{Im}(\alpha)=\left\{x_{1}, x_{2}, \ldots, x_{n}\right\}$ constructed in Note 1.8. Let $\alpha_{1}, \ldots, \alpha_{k} \in \mathcal{C}(k \in$ $\mathbb{N}$ ) be such that $\alpha=\alpha_{1} \cdots \alpha_{k}$. Since $J_{f} \cap\langle J\rangle=\emptyset$ and $\alpha \in J_{f}$, we must have $\alpha_{i} \notin J$, for some $i \in\{1, \ldots, k\}$. Hence, $n=|\operatorname{Im}(\alpha)| \leq\left|\operatorname{Im}\left(\alpha_{i}\right)\right|<\aleph_{0}$ (check the proof of Note 1.3). Thus, as $n \in \mathbb{N}$ is arbitrary, also in this case, $\mathcal{C}$ must contain an infinite number of elements of $J_{f}$.

Therefore, $\operatorname{rank}(\mathcal{O}(X): J) \geq \aleph_{0}$, as required.

Recall that, for $X \in\{\mathbb{Z}, \mathbb{Q}\}$, with the usual order, we have $X^{0}=X$. Therefore, as an immediate consequence of the last theorem, we obtain:

Corollary 1.10. Let $X \in\{\mathbb{Z}, \mathbb{Q}\}$, with the usual order. Then $\mathcal{O}(X)=\langle J\rangle$.

Notice that, for the chain $X=\mathbb{\mathbb { Z }}$ defined in the beginning of this section, we have $X^{0}=\{0\}$, whence also in this case $\mathcal{O}(X)=\langle J\rangle$.

On the contrary, we have: 
Proposition 1.11. With the usual order of $\mathbb{N}$, we have $\operatorname{rank}(\mathcal{O}(\mathbb{N}): J)=$ $\aleph_{0}$.

Proof. We already observed that $X^{0}=\emptyset$, for $X=\mathbb{N}$ equipped with the usual order. Hence, by Theorem [1.9, we obtain $\operatorname{rank}(\mathcal{O}(\mathbb{N}): J) \geq \aleph_{0}$. On the other hand, since $J_{f}=\mathcal{O}(\mathbb{N}) \backslash J$, we have $\operatorname{rank}(\mathcal{O}(\mathbb{N}): J) \leq\left|J_{f}\right|$. Therefore, this result follows by showing that $\left|J_{f}\right|=\aleph_{0}$. In fact, for each $n \in \mathbb{N}$ and each fixed subset $\left\{x_{1}, \ldots, x_{n}\right\}$ of $\mathbb{N}$ with $n$ elements, we have a bijection between the set $\left\{\alpha \in \mathcal{O}(\mathbb{N}) \mid \operatorname{Im}(\alpha)=\left\{x_{1}, \ldots, x_{n}\right\}\right\}$ and the set $\mathcal{P}_{n-1}(\mathbb{N} \backslash\{1\})$ of all subsets of $\mathbb{N} \backslash\{1\}$ with $n-1$ elements, namely $\alpha \longmapsto\left\{\min x_{i} \alpha^{-1} \mid i=2, \ldots, n\right\}$. Thus, since the set $\mathcal{P}_{f}(\mathbb{N})$ of all finite subsets of $\mathbb{N}$ has cardinal $\aleph_{0}, J_{f}$ is an infinite countable union of infinite countable sets and so $\left|J_{f}\right|=\aleph_{0}$, as required.

Observe that our Main Lemma gives us a necessary condition for having $\mathcal{O}(X)=\langle J\rangle$, namely $X^{0} \neq \emptyset$. We finish this note by presenting a sufficient condition:

Theorem 1.12. Let $X$ be an infinite chain such that $X \backslash X^{0}$ is finite. Then $\mathcal{O}(X)=\langle J\rangle$.

Proof. Notice that, $X^{-}$and $X^{+}$are both finite sets and $\left|X^{0}\right|=|X|$.

Take $\alpha \in \mathcal{O}(X)$.

First, suppose that $X^{0} \alpha \cap X^{0} \neq \emptyset$. Fix $u, v \in X^{0}$ such that $u \alpha=v$. If $u \leq v$, we define transformations $\alpha_{1}$ and $\alpha_{2}$ of $\mathcal{O}(X)$ by

$$
x \alpha_{1}=\left\{\begin{array}{ll}
x, & x<u \\
x \alpha, & u \leq x
\end{array} \text { and } x \alpha_{2}= \begin{cases}x \alpha, & x<u \\
v, & u \leq x<v \\
x, & v \leq x\end{cases}\right.
$$

On the other hand, if $v<u$, we define transformations $\alpha_{1}$ and $\alpha_{2}$ of $\mathcal{O}(X)$ by

$$
x \alpha_{1}=\left\{\begin{array}{ll}
x \alpha, & x \leq u \\
x, & u<x
\end{array} \text { and } x \alpha_{2}= \begin{cases}x, & x<v \\
v, & v \leq x<u \\
x \alpha, & u \leq x\end{cases}\right.
$$

It is a routine matter to show that both cases satisfy $\alpha_{1}, \alpha_{2} \in J$ and $\alpha=\alpha_{1} \alpha_{2}$. Then $\alpha \in\langle J\rangle$.

On the other hand, suppose that $X^{0} \alpha \cap X^{0}=\emptyset$. Then $\alpha \in J_{f}$ and so, by our Main Lemma, we obtain again $\alpha \in\langle J\rangle$, as required.

Clearly, the converse of this property is not valid in general, as the example $X=\overrightarrow{\mathbb{Z}}$ shows. Nevertheless, as an immediate application, for the usual chain of real numbers, we have: 
Corollary 1.13. With the usual order of $\mathbb{R}$, we have $\mathcal{O}(\mathbb{R})=\langle J\rangle$.

\section{References}

[1] M.E. Adams and M. Gould, Posets whose monoids of order-preserving maps are regular, Order 6 (1989), 195-201.

[2] A.Ya. Aizenštat, Regular semigroups of endomorphisms of ordered sets, Leningrad. Gos. Ped. Inst. Učen. Zap. 387 (1968), 3-11 (Russian).

[3] V.H. Fernandes, P. Honyam, T.M. Quinteiro and B. Singha, On semigroups of endomorphisms of a chain with restricted range, Semigroup Forum 89 (2014), 77-104.

[4] P.M. Higgins, J.D. Mitchell and N. Ruškuc, Generating the full transformation semigroup using order preserving mappings, Glasgow Math. J. 45 (2003), 557-566.

[5] J.M. Howie, Fundamentals of Semigroup Theory, Oxford, Oxford University Press, 1995.

[6] J.M. Howie, N. Ruškuc and P.M. Higgins, On relative ranks of full transformation semigroups, Comm. Algebra 26 (1998), 733-748.

[7] W. Mora and Y. Kemprasit, Regular elements of some order-preserving transformation semigroups, Int. J. Algebra 4 (2010), no. 13-16, 631-641.

[8] N. Ruškuc, On the rank of completely 0-simple semigroups, Math. Proc. Cambridge Philos. Soc. 116 (1994), 325-338.

Ilinka Dimitrova, Faculty of Mathematics and Natural Science, South-West University "Neofit Rilski", 2700 Blagoevgrad, Bulgaria; email: ilinka_dimitrova@swu.bg

Vítor H. Fernandes, CMA, Departamento de Matemática, Faculdade de Ciências e Tecnologia, Universidade NOVA de Lisboa, Monte da Caparica, 2829-516 Caparica, Portugal; e-mail: vhf@fct.unl.pt

JÖRG KopPITZ, Institute of Mathematics, University of Potsdam, 14469 Potsdam, Germany; email: koppitz@uni-potsdam.de 